\section{Revista Facultad 2020 de Ciencias Básicas Vol. 16(1) \\ enero-junio}

- ISSN: 1900-4699 - ISSN-e: 2500-5316 - pp. 69-78

DOI: https://doi.org/10.18359/rfcb.4710

(c) $(1)(9)$

\title{
Recursos florales utilizados por el abejorro nativo Bombus atratus (Hymenoptera: Apidae) bajo condiciones de invernadero y campo abierto en la Sabana de Bogotá, Colombia*
}

Sandy Carolina Padilla Báez ${ }^{\mathrm{a}}$ - Cláudia Inês da Silva ${ }^{\mathrm{b}}$ - José Ricardo Cure Hakim

Resumen: El abejorro nativo Bombus atratus es un importante polinizador de cultivos de interés agricola. El estudio de su biología y su cría en cautiverio ha sido el tema de investigación de nuestro grupo durante más de una década. Hasta el momento se desconocían las fuentes de recursos de alimento usadas por las colonias criadas bajo el protocolo del grupo de investigación, motivo por el cual se llevó a cabo el presente trabajo. Se ubicaron colonias en invernadero y en campo abierto. Se colectó un total de 105 cargas polínicas provenientes de corbículas de obreras: 85 de la condición de invernadero y veinte de la condición de campo abierto, las cuales se procesaron mediante el método de acetólisis ácida. Se identificaron 36 tipos polínicos de los cuales quince se denominaron como importantes fuentes de polen y néctar en la dieta de $B$. atratus. El $42 \%$ de la dieta se compone de especies de la familia Solanaceae, el 26 \% de la familia Myrtaceae y el $25 \%$ de dieferentes especies de arvenses. Las colonias ubicadas tanto en campo abierto como en invernadero comparten la mayoría de las fuentes de recursos florales, sin embargo, algunos recursos fueron explotados más que otros debido a su disponibilidad en cada área. Los recursos florales que se encontraron con mayor frecuencia dentro de las cargas polínicas fueron: Brassica rapa L., Raphanus sativus L., Trifolium repens L., Trifolium pratense L., Eucalyptus globulus Labill, Solanum quitoense Lam. y Solanum lycopersicum L.

Palabras clave: cría de abejorros; palinología; cargas polínicas; polinización; interacción abeja-planta

* Artículo de investigación.

a Magíster en Biología. Bióloga, Universidad Militar Nueva Granada, Colombia. Facultad de Ciencias Bási-

cas y Aplicadas, Universidad Militar Nueva Granada. Bogotá D. C., Colombia.

Correo electrónico: sandycarolinapadilla@gmail.com

ORCID: https://orcid.org/0000-0003-1820-4936

b Doctora en Ecología y Conservación de Recursos Naturales por la Universidade Federal de Uberlândia. Universidade Federal de São Carlos, Sorocaba, Brasil.

Correo electrónico: claudiainess@usp.br

ORCID: https://orcid.org/0000-0003-0836-8662

c Doctor en Ciencias Biológicas. Universidad Militar Nueva Granada, Bogotá, Colombia.

Correo electrónico: jose.cure@unimilitar.edu.co

ORCID: https://orcid.org/0000-0001-5816-0259 
Recibido: $04 / 04 / 2020$

Aceptado: 13/08/2020

Disponible en linea: 19/03/2021

Cómo citar: S. C. Padilla Báez, C. I. Da Silva, y J. R. Cure Hakim, «Recursos florales utilizados por el abejorro nativo Bombus atratus (Hymenoptera: Apidae) bajo condiciones de invernadero y campo abierto en la Sabana de Bogotá, Colombia», Rev. Fac. Cienc. Básicas, vol. 16, n. ${ }^{0}$ 1, mar. 2021.

\title{
Flower resources used by the native Bombus atratus (Hymenoptera: Apidae) bumblebee under greenhouse and open field conditions in the Sabana de Bogotá, Colombia
}

\begin{abstract}
Bombus atratus native bumblebee is an important pollinator of agricultural interest crops. The study of their biology and their breeding in captivity has been the research topic of our group for more than a decade. Until now, the sources of food resources used by the colonies raised under the protocol of the research group were unknown, which is why this work was carried out. Colonies were located in the greenhouse and in the open field. A total of 105 pollen loads from worker corbiculae were collected: 85 from the greenhouse condition and twenty from the open field condition, which were processed using the acid acetolysis method. Thirty-six pollen types were identified, fifteen of which were named as important sources of pollen and nectar in the diet of B. atratus. $42 \%$ of the diet is made up of species of the Solanaceae family, $26 \%$ of the Myrtaceae family and $25 \%$ of different species of arvenses. The colonies located both in the open field and in the greenhouse share most of the sources of flower resources, however, some resources were more exploited than others due to their availability in each area. The flower resources that were most frequently found within the pollen loads were: Brassica rapa L., Raphanus sativus L., Trifolium repens L., Trifolium pratense L., Eucalyptus globulus Labill, Solanum quitoense Lam. y Solanum lycopersicum L.
\end{abstract}

Keywords: bumblebee keeping; palynology; pollen loads; pollination; bee-plant interaction

\section{Recursos florais utilizados pela mamangava nativa Bombus atratus (Hymenoptera: Apidae) sob condições de estufa e campo aberto na savana de Bogotá, Colômbia}

Resumo: a mamangava nativa Bombus atratus é um importante polinizador de cultivos com fins agrícolas. O estudo de sua biologia e sua criação em cativeiro vem sendo tema de pesquisa de nosso grupo durante mais de uma década. Até momento, eram desconhecidas as fontes de recursos de alimentos usadas pelas colônias criadas sob o protocolo do grupo de pesquisa, razão pela qual foi realizado este trabalho. Foram localizadas colônias em estufa e em campo aberto. Foram coletadas 105 cargas polínicas provenientes de corbículas de operárias: 85 na condição de estufa e 20 na condição de campo aberto, as quais foram processadas mediante o método de acetólise ácida. Foram identificados 36 tipos polínicos, dos quais 15 se denominaram importantes fontes de pólen e néctar na dieta de $B$. atratus. $42 \%$ da dieta está composta de espécies da família Solanaceae; $26 \%$ da família Myrtaceae e 25 \% de diferentes espécies de arvenses. Tanto as colônias localizadas em campo aberto quanto em estufa compartilham a maioria das fontes de recursos florais; contudo, alguns recursos foram explorados mais do que outros devido à sua disponibilidade em cada área. Os recursos florais que foram encontrados com maior frequência dentro das cargas polínicas foram: Brassica rapa L., Raphanus sativus L., Trifolium repens L., Trifolium pratense L., Eucalyptus globulus Labill, Solanum quitoense Lam. e Solanum lycopersicum L.

Palavras-chave: criação de mamangava; palinologia; cargas polínicas; polinização; interação abelha-planta 


\section{Introducción}

La polinización es un servicio de soporte del ecosistema que es necesario para la fecundación de aproximadamente el $90 \%$ de las angiospermas del planeta, muchas de las cuales son económicamente importantes en la agricultura [1], [2]. Actualmente existe una preocupación mundial debido a que se ha reportado una paulatina reducción de las poblaciones de muchas especies de abejas [3], [4], [5], lo que ha promovido iniciativas internacionales como, por ejemplo, la Iniciativa Internacional de Polinizadores (IPI), la Intergovernmental Science-Policy Platform on Biodiversity and Ecosystem Services (Ipbes), o la Plataforma Brasileira de Biodiversidade e Serviços Ecossistêmicos BPBES, así como otras de carácter nacional como la Iniciativa Colombiana de Polinizadores con énfasis en abejas (ICPA), con el fin de desarrollar estrategias orientadas a mitigar dicha reducción [6].

Adicionalmente, se ha dado especial interés al uso de especies nativas para la polinización de cultivos, evitando la modificación de la dinámica de la polinización en ambientes naturales y la dispersión de patógenos [7], [8]. Asimismo, permite aprovechar la oferta floral regional y favorecer la preservación de áreas silvestres alrededor o dentro de los monocultivos, lo que favorece la preservación de estos organismos.

Bombus (Fervidobombus) pauloensis Friese 1913, también conocida como Bombus (Fervidobombus) atratus Franklin 1913 [9], es un abejorro nativo que se distribuye desde la Cordillera Oriental de los Andes colombianos hasta Argentina, incluyendo Venezuela, Ecuador, Perú, Paraguay, Uruguay, Brazil y Bolivia, desde los 0 a los 3050 m. s. n. m.; presenta mayor densidad de población entre los 1800 y los 2800 m. s. n. m. [10], [11]. Comparada con A. mellifera, B. atratus presenta características morfológicas y fisiológicas que lo favorecen como polinizador de algunas especies de plantas; por ejemplo, tiene la glosa más larga, son de mayor tamaño, tienen la capacidad de vibrar en las anteras de las flores y son más tolerantes al frío. Lo anterior les permite visitar flores de corola profunda, cargar más polen, incrementar la probabilidad de hacer contacto de este con el estigma, facilitar la salida del polen de las anteras poricidas y forrajear incluso con lluvia o vientos fuertes [12], [13].
El grupo de investigación Biodiversidad y Ecología de Abejas Silvestres (BEAS) ha trabajado desde 1998 en la biología, en la ecología de la polinización y en la cría en cautiverio de la especie nativa $B$. atratus [14], lo que le ha permitido estandarizar un esquema de cría de colonias en cautiverio para su utilización en estudios de polinización de diferentes cultivos tales como lulo Solanum quitoense [15], tomate Solanum lycopersicum [16], uchuva Physalis peruviana [17], fresa Fragaria $x$ ananassa [18], mora Rubus silvestris [19] y pimentón Capsicum annuum [20]. Este esquema de cría permite iniciar colonias bajo condiciones de cautiverio, las cuales, posteriormente, son transferidas a condiciones de invernadero o espacios abiertos con el propósito de completar su ciclo hasta obtener machos y nuevas reinas (individuos sexuados). En estas condiciones se ofrecen diferentes fuentes de recursos florales a fin de que las obreras puedan forrajear libremente en busca de su alimento [21]. Con la finalidad de hacer una mejor selección de las plantas que deben ser ofrecidas en estas condiciones para la cría de las colonias, el objetivo del presente trabajo fue conocer los recursos florales utilizados en la dieta de B. atratus bajo el sistema de cría en condiciones de invernadero y campo abierto.

\section{Materiales y métodos}

\section{Área de estudio}

El trabajo se realizó en el campus de la Universidad Militar Nueva Granada (UNMG), localizado en Cajicá (Cundinamarca, Colombia), durante los meses de marzo, abril y mayo del 2013. Cajicá hace parte de la Sabana de Bogotá, está ubicado a una altura de 2580 m. s. n. m. y presenta una temperatura promedio anual de $14,0^{\circ} \mathrm{C}$ y una precipitación anual de $830 \mathrm{~mm}$. La vegetación del campus cuenta con plantas nativas y exóticas, de las cuales el $46 \%$ son cultivadas y las demás crecen espontáneamente. Las familias mas representativas del campus son Asteraceae, Leguminosae y Poaceae [22]. Dentro del campus se trabajó bajo dos condiciones: campo abierto $\left(04^{\circ} 56^{\prime} 43,8^{\prime \prime} \mathrm{N}\right.$ y $74^{\circ} 00^{\prime} 38,5^{\prime \prime}$ W) e invernadero Bombinario

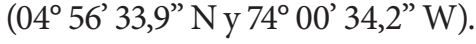


El área de campo abierto estaba, en su mayoría, cultivada con pasto kikuyo (Penisettum clandestinum) y trébol rojo (Trifolium pratense), sin poda, riego ni fertilización (figuras 1A y 1B).

El invernadero Bombinario es un espacio de $2000 \mathrm{~m}^{2}$ construido para el mantenimiento de colonias de B. atratus. Allí se les ofrece protección $y$ recursos florales permanentes para su alimentación (véanse las figuras 1c y 1D). Además de un invernadero cerrado, este espacio cuenta con un área externa anexa, delimitada y cultivada con varias especies florales. Durante el desarrollo del trabajo, el invernadero presentó floración de plantas de lulo (S. quitoense), trébol rojo (T. pratense), colza (Brassica rapa), rábano (Raphanus sativus), girasol (Helianthus annuus), dália (Dahlia imperialis) y guaba (Phytolacca bogotensis) como principales fuentes de polen y néctar para las colonias [21]. El invernadero Bombinario está rodeado por otros invernaderos de investigación donde se cultivan, principalmente, plantas como tomate (S. lycopersicum), fresa (Fragaria $x$ ananassa), arveja (Pisum sativum), lupino (Lupinus bogotensis) y maracuyá (Passiflora edulis), entre otras.

\section{Cría de colonias de $B$. atratus}

Se colectaron reinas de B. atratus en condiciones silvestres en la zona de la Sabana de Bogotá entre 5 y 20 $\mathrm{km}$ de distancia alrededor del campus. Las reinas se llevaron, posteriormente, a la cámara de cría en cautiverio del grupo BEAs. La cría en cautiverio se llevó a cabo de acuerdo con el protocolo desarrollado por el grupo de investigación, según el cual las colonias son alimentadas con polen colectado por $A$. mellifera y solución azucarada (proporción 1:1 p/v) [14], [23]. Con el fin de que las colonias alcanzaran la fase de producción de sexuados, las colonias fueron trasladadas a las condiciones de campo abierto y al invernadero Bombinario donde completaron su ciclo. Bajo estas condiciones, las obreras podían forrajear libremente y así colectar el polen y el néctar necesarios para alimentar la cría. Las colonias ubicadas dentro del invernadero tenían acceso a los recursos que se encontraban tanto adentro como afuera del invernadero, debido a la implementación de dos salidas (piqueras) que les permitían a las obreras forrajear dentro del invernadero y fuera de este (véase la Figura 1D). Se colocaron cinco colonias en la condición de campo abierto y ocho colonias en la condición de invernadero.

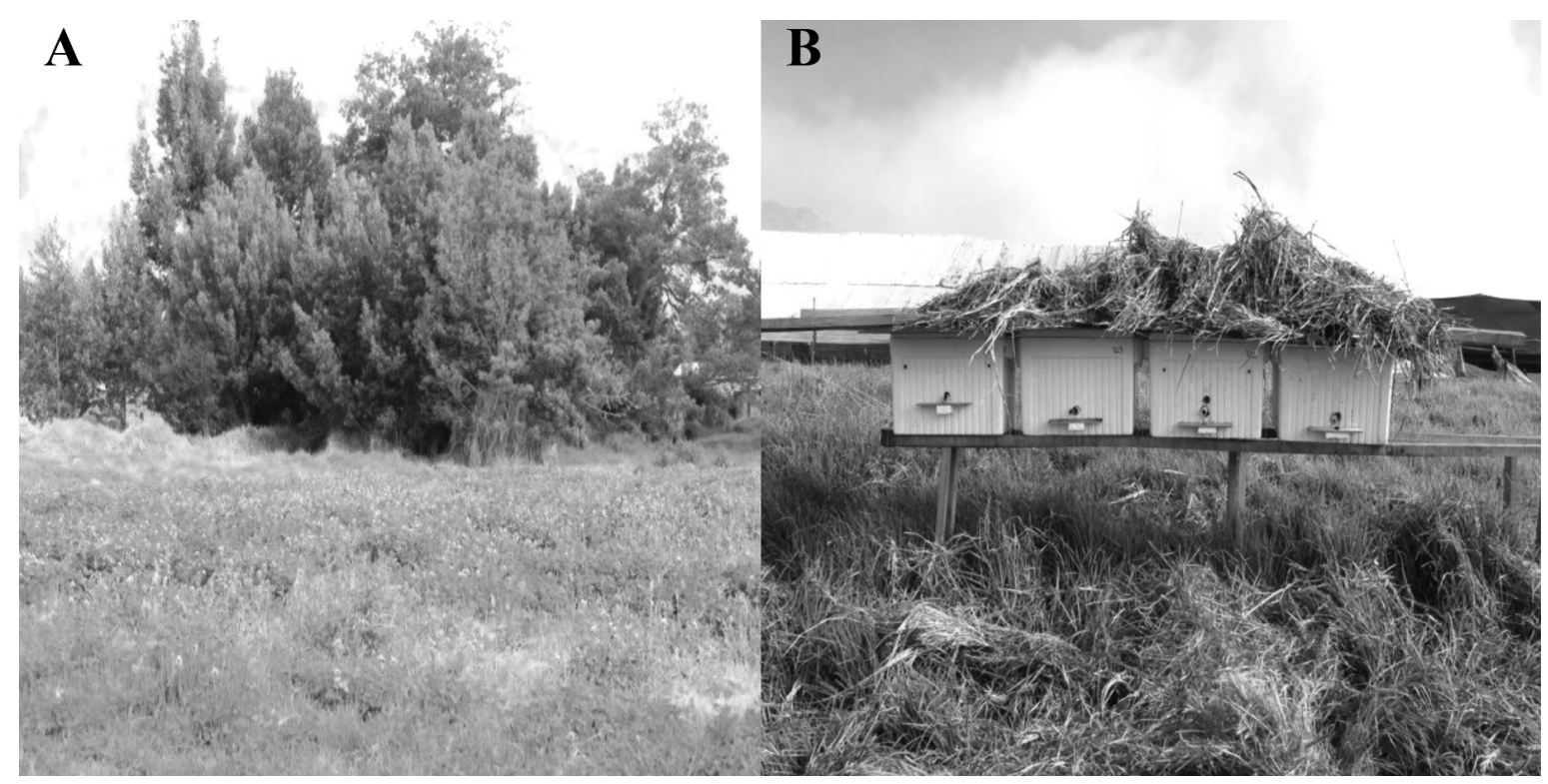




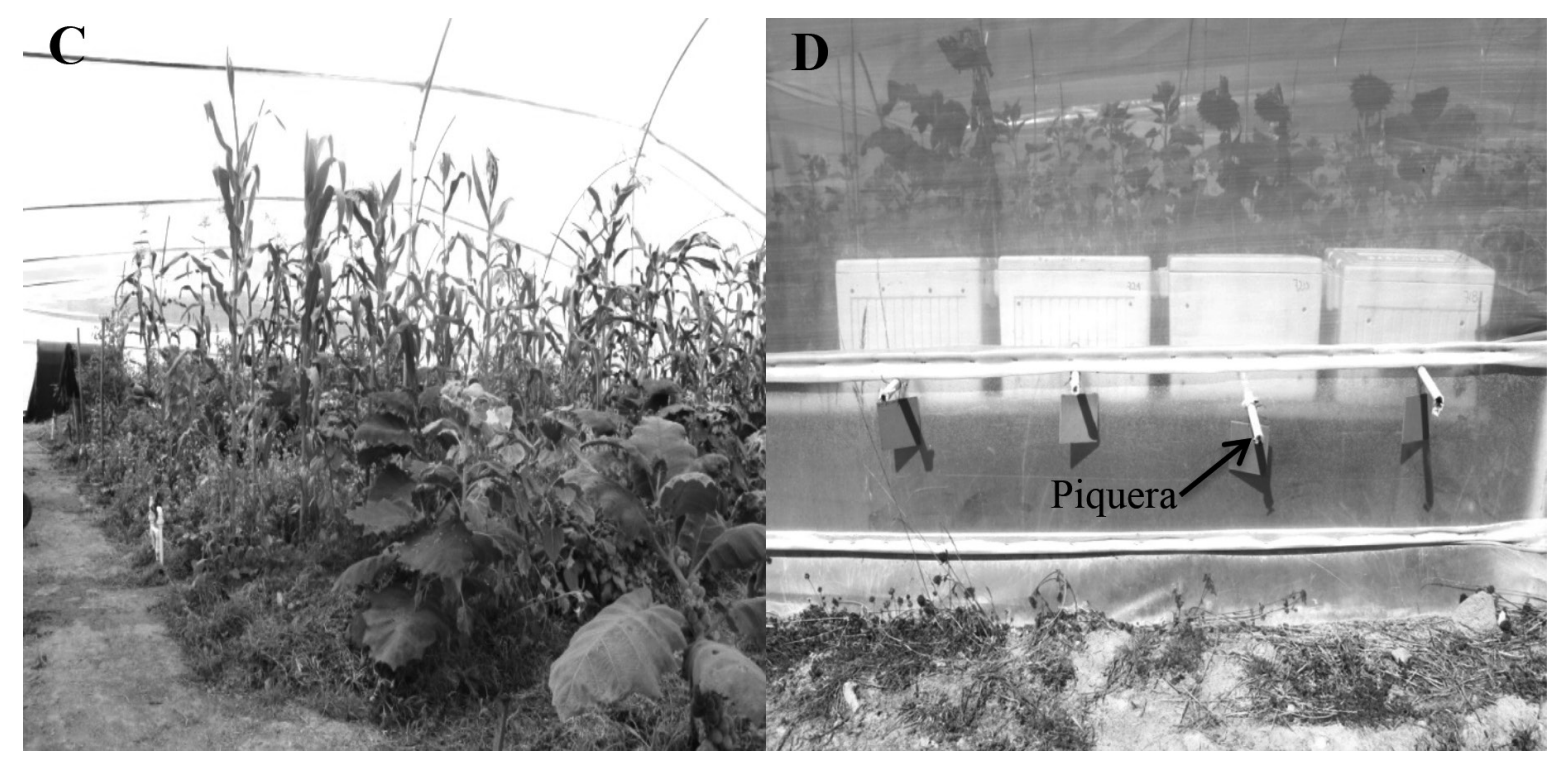

Figura 1. Áreas de estudio en el campus de la Universidad Militar Nueva Granada. Condición de campo abierto indicando la disposición de las colonias (A y B). Condición de invernadero (Bombinario) indicando la disposición de la piquera doble que da acceso a forrajear adentro y afuera del invernadero (C y D).

Fuente: elaboración propia.

\section{Recursos florales usados en la dieta de $B$. atratus}

Durante el periodo de estudio se realizó una colecta de anteras de treinta plantas en floración que se encontraban adentro y alrededor del invernadero Bombinario. Se construyó un catálogo polínico digital de las plantas colectadas siguiendo la técnica de acetólisis descrita por Erdtman [24], con las modificaciones propuestas por Silva et al. [25] para la extracción de polen y la preparación de láminas permanentes. El catálogo polínico digital se construyó a partir de fotografías de los granos de polen en vista polar, ecuatorial, y de la ornamentación de la exina de cada una de las especies de plantas que sirvió como referencia para la posterior identificación por comparación de las cargas polínicas de las abejas [25]. Estas láminas actualmente se encuentran almacenadas en la colección palinológica del grupo de investigación Biodiversidad y Ecología de Abejas Silvestres (PBEAs) del Laboratorio de Entomología del Campus de la UMNG en Cajicá, Colombia. La información de la palinoteca está disponible en la Red de Catálogos Polínicos online (RCPOl) [26].
Se colectó un total de 105 cargas polínicas provenientes de las corbículas de obreras presentes dentro de las colonias ubicadas en la condición de invernadero $(n=85)$ y de las colonias ubicadas en la condición de campo abierto $(n=20)$. Las muestras de polen se procesaron y montaron siguiendo el protocolo descrito [24], [25]. Se realizaron láminas para las muestras polínicas de cada individuo colectado. La identificación de cargas polínicas de los visitantes florales se realizó mediante la comparación con el catálogo polínico de referencia preparado con las plantas presentes en el área de estudio. Las láminas con las cargas polínicas de las abejas fueron depositadas igualmente en la palinoteca del Grupo de Investigación de Biodiversidad y Ecología de Abejas Silvestres (PBEAs) del campus de la UMNG.

Para cada una de las muestras con cargas polínicas se realizó un análisis cualitativo con el fin de conocer la riqueza de las especies de plantas visitadas y se complementó con un análisis cuantitativo en el que se identificaron los primeros cuatrocientos granos de cada muestra para determinar la frecuencia de los tipos polínicos [27]. Cada tipo polínico se clasificó de acuerdo con Maurizio y Louveaux [28]: 
polen dominante (PD $>45 \%$ del total de granos), polen accesorio (PA entre 15 y $45 \%$ ), polen aislado importante (PAI entre 3 y $14 \%$ ), polen aislado ocasional (PAO entre 1 y $3 \%$ ) y polen presente $(\mathrm{PP}<1 \%$ ). Se elaboró una tabla en la que se relacionó el conteo de polen de las diferentes especies de planta para cada una de las colonias evaluadas.

Adicionalmente, mediante el software PAST (v. 3.0) se realizó un análisis de diversidad por medio del índice Shannon-Wiener para cada condición y una prueba de chi cuadrado con el número total de plantas visitadas en la condición de invernadero y de campo abierto.

\section{Resultados y discusión}

Dentro de las 105 cargas polínicas procesadas, se identificaron 36 tipos polínicos distribuidos en quince familias, dos de ellos no identificados. De los 36 tipos polínicos identificados, 33 fueron observados en las colonias ubicadas en el invernadero Bombinario y 24 en campo abierto. El tipo polínico Taraxacum officinale incluye también la especie Hypochaeris radicata debido a que presentan una forma de grano muy parecida, lo que impide identificar con exactitud la especie de planta a la cual pertenece (Tabla 1).

Tabla 1. Frecuencia de los tipos polínicos encontrados en las cargas polínicas de las obreras en el invernadero y a campo abierto. Tipos polínicos importantes en la dieta de B. atratus: granos de polen dominantes (****PD), accesorios (***PA), aislados importantes (**PAI) y aislados ocasionales ( $\left.{ }^{*} P A O\right)$ [28].

\begin{tabular}{|c|c|c|c|c|c|}
\hline Familia & $\begin{array}{l}\text { Especie planta/tipo } \\
\text { polínico }\end{array}$ & Fuente & Origen & $\begin{array}{l}\text { Invernadero } \\
(\%)\end{array}$ & $\begin{array}{l}\text { Campo abierto } \\
(\%)\end{array}$ \\
\hline Anacardiaceae & Schinus sp. & No conocido & - & 0,023 & \\
\hline \multirow[t]{7}{*}{ Asteraceae } & Cirsium vulgare (Savi) Ten. & Polen/néctar & Exótica & 0,5 & 0,03 \\
\hline & $\begin{array}{l}\text { Dahlia imperialis Roezl ex } \\
\text { Ortgies }\end{array}$ & Polen/néctar & Exótica & 0,05 & 0,01 \\
\hline & Helianthus annuus L. & Polen/néctar & Exótica & $5,61 * *$ & \\
\hline & Senecio sp. & No conocido & - & 0,06 & \\
\hline & $\begin{array}{l}\text { Tithonia diversifolia } \\
\text { (HemsI.) A.Gray }\end{array}$ & Polen/néctar & Nativa & 0,06 & 0,03 \\
\hline & sp1 & No conocido & - & 0,01 & \\
\hline & $\begin{array}{l}\text { Tipo Taraxacum officinale } \\
\text { (L.) Weber ex F.H.Wigg. }\end{array}$ & Polen/néctar & Exótica & $2,3 *$ & 0,06 \\
\hline Bignoniaceae & $\begin{array}{l}\text { Tecoma stans (L.) Juss. ex } \\
\text { Kunth }\end{array}$ & Néctar & Exótica & 0,05 & \\
\hline \multirow[t]{2}{*}{ Brassicaceae } & Brassica rapa L. & Polen/néctar & Exótica & $4,77 * *$ & $8,39 * *$ \\
\hline & Raphanus sativus L. & Polen/néctar & Exótica & $1,67 *$ & $7,68 * *$ \\
\hline Ericaceae & Vaccinium corymbosum L. & Polen/néctar & Exótica & 0,003 & \\
\hline \multirow[t]{5}{*}{ Leguminosae } & Lupinus bogotensis Benth. & Polen/néctar & Nativa & 0,02 & 0,6 \\
\hline & Acacia decurrens Willd. & Polen & Exótica & & 0,1 \\
\hline & Pisum sativum L. & Polen/néctar & Exótica & & 0,15 \\
\hline & $\begin{array}{l}\text { Senna multiglandulosa } \\
\text { (Jacq.) H.S.Irwin \& Barneby }\end{array}$ & Polen & Exótica & 0,11 & $3,88 * *$ \\
\hline & $\begin{array}{l}\text { Senna viarum (Little) } \\
\text { H.S.Irwin \& Barneby }\end{array}$ & Polen & Nativa & 0,02 & 0,08 \\
\hline
\end{tabular}




\begin{tabular}{|c|c|c|c|c|c|}
\hline & Trifolium pratense L. & Polen/néctar & Exótica & 10,16 ** & $14,00 * *$ \\
\hline & Trifolium repens $\mathrm{L}$. & Polen/néctar & Exótica & 0,27 & $1,48 *$ \\
\hline & Senna sp1 & Polen & - & $2,17^{*}$ & $1,90 *$ \\
\hline Malvaceae & Abutilon insigne Planch. & Polen/néctar & Exótica & 0,02 & 0,01 \\
\hline \multirow[t]{2}{*}{ Myrtaceae } & $\begin{array}{l}\text { Syzygium paniculatum } \\
\text { Gaertn. }\end{array}$ & Polen/néctar & Exótica & 0,71 & \\
\hline & Eucalyptus globulus Labill. & Polen/néctar & Exótica & $24,78 * * *$ & $16,66 * * *$ \\
\hline Oxalidaceae & Oxalis stricta L. & Polen/néctar & Exótica & 0,05 & \\
\hline Passifloraceae & Passiflora edulis Sims & Polen/néctar & Exótica & 0,01 & 0,01 \\
\hline Poaceae & Zea mays L. & Polen & Exótica & & 0,03 \\
\hline Plantaginaceae & Digitalis purpurea L. & Polen/néctar & Exótica & 0,06 & \\
\hline Polygonaceae & Polygonum segetum Kunth & Polen/néctar & Exótica & $1,30 *$ & 0,5 \\
\hline \multirow[t]{3}{*}{ Rosaceae } & $\begin{array}{l}\text { Fragaria } \mathrm{x} \text { ananassa } \\
\text { (Duchesne ex Weston) } \\
\text { Duchesne ex Rozier }\end{array}$ & Polen/néctar & Exótica & 0,02 & \\
\hline & $\begin{array}{l}\text { Cotoneaster pannosus } \\
\text { Franch. }\end{array}$ & Polen & Exótica & $2,18 *$ & $2,06 *$ \\
\hline & Rosa sp. & Polen/néctar & - & $2,01 *$ & \\
\hline \multirow[t]{3}{*}{ Solanaceae } & Solanum quitoense Lam. & Polen & Nativa & $9,65 * *$ & $16,55^{* * *}$ \\
\hline & Solanum sp1 & Polen & - & $1,39 *$ & 0,75 \\
\hline & Solanum lycopersicum L. & Polen & Exótica & $29,65 * * *$ & $24,66 * * *$ \\
\hline \multirow[t]{2}{*}{ Indeterminados } & sp1 & No conocido & - & 0,18 & 0,38 \\
\hline & sp2 & No conocido & - & 0,13 & \\
\hline \multicolumn{4}{|c|}{ Total general de tipos polínicos } & 33 & 24 \\
\hline
\end{tabular}

Fuente: elaboración propia.

Las familias Solanaceae (42\%), Myrtaceae (26\%) y Leguminosae (13\%) comprenden el $80 \%$ de las fuentes de recursos florales usados por B. atratus, de manera que son $S$. lycopersicum y $S$. quitoense las fuentes más importantes de polen tanto en invernadero como en campo abierto (véase la Figura 2A). Las dos condiciones presentan una diversidad de tipos polínicos similar ( $\mathrm{H}$ invernadero: 2,128; $\mathrm{H}$ campo abierto: 2,117). Se observó que las colonias de invernadero y campo abierto colectan porcentajes similares de polen de las mismas familias de plantas (véase la Figura 2B). No se encontró diferencia significativa en el número de plantas visitadas entre los tratamientos, lo que sugiere que $B$. atratus puede criarse tanto en invernadero como en campo abierto ( $x$-squared $=14,211, d f=1, p$-value: 0,2332$)$.
El $21 \%$ de las especies visitadas en el invernadero y el $33 \%$ de las visitadas en el campo abierto son arvenses exóticas: T. repens, T. pratense, $R$. sativus, B. rapa, tipo T. officinale y $P$. segetum. Lo anterior muestra la adaptación de $B$. atratus a ambientes altamente intervenidos como los que se encuentran alrededor del área de estudio. Estos recursos suplen gran parte de la dieta de estos abejorros en zonas intervenidas o urbanas debido a que son especies de arvenses que se encuentran con frecuencia en zonas de pastizal [29]. Asimismo, las especies T. repens y $T$. pratense han sido reportadas como un recurso de polen y néctar importante para diferentes especies del género Bombus tanto en diferentes países de Europa (Bombus agrorum, Bombus lucorum, Bombus hortorum, Bombus pascuorum) 
como en zonas altoandinas de Colombia (Bombus hortulanus y Bombus funebris) [30], [31], [32].

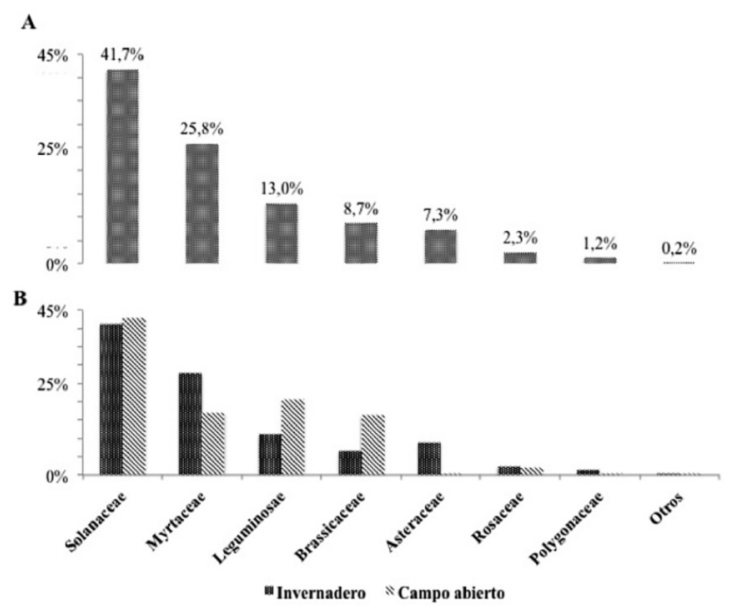

Figura 2. Porcentajes relativos de pólen de las principales familias de plantas visitadas por B. atratus. (A) Totales. (B) Según la localización de las colonias.

Fuente: elaboración propia.

Eucalyptus globulus es la principal especie de la familia Myrtaceae visitada por B. atratus; se encuentra en una plantación a $300 \mathrm{~m}$ de distancia de las colonias ubicadas en el invernadero y a cerca de $15 \mathrm{~m}$ de las colonias situadas en campo abierto. Por lo anterior, con el fin de ofrecer fuentes de recursos dirigidas a la alimentación de las colonias de $B$. atratus para su cría en el invernadero, se sugiere que se permita el acceso a la plantación de eucalipto aledaño, lo que indica necesariamente una salida al exterior mediante la implementación de la doble piquera.

Adicionalmente, se sugiere que se mantenga una oferta constante de especies de la familia Solanaceae debido a que cuenta con un alto contenido proteico [33], [34], [35]. Esta característica hace que estos recursos sean muy apetecidos por los abejorros, ya que ellos, a diferencia de A. mellifera, prefieren polen que cuente con un mayor contenido proteico y más aminoácidos esenciales, aunque estén en menor cantidad [36]. Las plantas, por su parte, también se ven beneficiadas por las visitas de las obreras, ya que algunas de las especies de la familia Solanaceae se caracterizan por tener anteras poricidas y necesitan de la vibración de las obreras o reinas para la extracción del polen y su correcta polinización [37].

Para complementar la dieta es recomendable incluir dentro de los recursos ofrecidos otras especies de plantas que presenten polen con alto contenido proteico, como, por ejemplo, B. officinalis [35], así como otras especies de la familia Leguminosae, preferiblemente nativas como lo son L. bogotensis y S. multiglandulosa.

En estudios realizados en nidos silvestres de $B$. atratus en Argentina se ha reportado que dentro de las fuentes de recursos más importantes en la dieta para esta especie se destacan Eucalyptus spp. como fuente de néctar y Solanum spp. como fuente de polen, lo cual coincide con lo encontrado en este estudio [38]. Otras especies de plantas de las familias Solanaceae, Myrtaceae y Leguminosae también han sido reportadas como recursos importantes para $B$. atratus y otras especies de Bombus. Con respecto a B. atratus y Bombus morio en Brasil se destacan Solanum paniculatum y Psisium guajava [39], y para $B$. agrorum y B. lucorum en Escocia se reportan Lotus corniculatus y Vicia sp. [30], [40], [41].

El presente trabajo da a conocer las especies de plantas que mantienen las colonias de B. atratus en las condiciones de cría de campo abierto y el invernadero Bombinario, así como sugiere la implementación de otras especies de plantas para complementar la dieta de las colonias bajo estas condiciones. Se concluye que las especies de las familias Solanaceae y Leguminosae proporcionan a estas colonias su principal fuente de polen, y el acceso al bosque de eucalipto es una fuente primordial de polen y néctar. Asimismo, las arvenses presentes en el campus complementan favorablemente la dieta de las colonias.

\section{Agradecimientos}

A la Vicerrectoría de Investigaciones de la Universidad Militar Nueva Granada por la financiación del trabajo mediante el proyecto cias 1463, y a la RCPol-Redes de Catálogos Polínicos online (FDTE process\#001505) por el apoyo en la identificación del polen. 


\section{Referencias}

[1] C. Kremen y R. S. Ostfeld, "A call to ecologists: measuring, analyzing and managing ecosystem services", Front. Ecol. Environ., vol. 3, n. ${ }^{\circ}$ 10, pp. 540-548, 2005.

[2] N.O. M. Waser y J. Ollerton, Plant-Pollinator interactions. From specialization to generalization. Chicago: The University of Chicago Press, 2006.

[3] C. Carvell, D. Roy, S. Smart, R. Pywell, C. Preston y D. Goulson, "Declines in forage availability for bumblebees at a national scale", Biol. Conserv., vol. 132, pp. 48-489, 2006.

[4] S. Colla y L. Packer, "Evidence for decline in eastern North American bumblebees (Hymenoptera: Apidae), with special focus on Bombus affinis Cresson", Bio. Conserv., vol. 17, pp. 1379-1391, 2008.

[5] D. Goulson, G. C. Lye y B. Darvill, "Decline and conservation of bumble bees", Ann. Rev. Entomol., vol. 53, pp. 191-208, 2008.

[6] Convention on Biological Diversity, Conference of the parties cop 5. Decision v/5. II: international initiative for the conservation and sustainable use of pollinators, 2000. [En línea]. Disponible en: http:// www.cbd.int/decision/cop/?id=7147.

[7] K. Winter et al., Importation of non-native bumble bees into North America: potential consequences of using Bombus terrestris and other non-native bumble bees for greenhouse crop pollination in Canada, Mexico and the United States. San Francisco: North American Pollinator Protection Campaign (NAPPC), 2006.

[8] C. Morales, M. Arbetman, S. Cameron y M. Aizen, "Ecological replacement of a native bumble bee by invasive species”, Front. Ecol. Environ., vol. 11, pp. 529-534, 2013.

[9] J. S. Moure y G. A. R. Melo, "Bombini Latreille, 1802”, En Catalogue of Bees (Hymenoptera, Apoidea) in the neotropical region-online version, J. S. Moure, D. Urban y G. A. R. Melo, Eds. Curiitiba, Brasil: Sociedade Brasileira de Entomologia, 2012. [En línea]. Disponible en: http://www.moure.cria.org.br/catalogue

[10] A. Liévano, R. Ospina y G. Nates, "Contribución al crecimiento de la taxonomía del género Bombus en Colombia (Hymenoptera: Apidae)", Trianea. Acta Cient. Tecno. Inder., vol. 5, pp. 221-233, 1994.

[11] A. Abrahamovich y N. O. Díaz, Bumble bees of the neotropical region (Hymenoptera: Apidae)", Biota Colomb., vol. 3, n. ${ }^{\circ}$ 2, pp. 199-214, 2002.
[12] S, Corbet, I. Williams y J. Osborne, "Bees and the pollination of crops and wild flowers in the European community", Bee World, vol. 72, pp. 47-59, 1991.

[13] K. Delaplane y D. Mayer, Crop pollination by bees. Londres: CABI publishing, 2000.

[14] P. Cruz, M. T. Almanza y J. R. Cure, "Logros y perspectivas de la cría de abejorros del genero Bombus en Colombia”, Rev. Facul. Cienc. Bás., vol. 3, pp. 49-60, 2007.

[15] M. T. Almanza, "Management of Bombus atratus bumblebees to pollinate Lulo (Solanum quitoense L.), a native fruit from the Andes of Colombia”, tesis doctoral, Cuvillier Verlag Gottingen, Germany, 2007.

[16] J. Aldana, R. J. Cure, M. T Almanza, D. Vecil y D. Rodriguez, "Efecto de Bombus atratus (Hymenoptera: Apidae) sobre la productividad de tomate (Lycopersicon esculentum Mill.) bajo invernadero en la Sabana de Bogotá, Colombia", Agron. Colomb., vol. 25, n. ${ }^{\circ}$ 1, pp. 62-72, 2007.

[17] L. Camelo, L. Díaz, J. R. Cure y M. T. Almanza, "Morfología floral de la uchuva y comportamiento de visitas de la especie de abejorros Bombus atratus (Hymenoptera: Apidae) bajo invernadero", En resúmenes del XXXI Cong. Soc. Colomb. Entomol. Socolen, Bogotá, Colombia, 2004, pp. 86.

[18] C.A. Poveda, D. Riaño, L. Aguilar y J.R. Cure, “Eficiencia de polinización de colonias huérfanas de Bombus atratus (Hymenoptera: Apidae) en fresa (Fragaria x ananassa) bajo cubierta”, Acta Biol. Colomb., vol. 23, n. ${ }^{\circ}$ 1, pp. 73-79, 2018, doi: http://dx.doi. org/10.15446/abc.v23n1.61648

[19] J. Zuluaga, "Evaluación de la actividad polinizadora de Bombus atratus (Hymenoptera: Apidae) en un cultivo de mora de castilla (Rubus glaucus)", tesis de grado, Facultad de Ciencias Básicas y Aplicadas, Universidad Militar Nueva Granada, 2011.

[20] D. Riaño, J. Pacateque, J. R. Cure y D. Rodríguez, "Comportamiento y eficiencia de polinización de Bombus atratus Franklin en pimentón (Capsicum annun L.) sembrado bajo invernadero", Rev. Colomb. Cienc. Hort., vol. 9, n. ${ }^{\circ}$ 2, pp. 259-267, 2015, doi: http://dx.doi.org/10.17584/rcch.2015v9i2.4182

[21] E. Romero, C. Pinilla, J. R., Cure, D. Riaño, S. Padilla y M. Aguilar, "Desarrollo de un escenario de campo para el estudio de especies nativas de abejorros (Bombus spp.) de los Andes colombianos (Hymenoptera: Apidae)", Rev. Facul. Cien. Bás., vol. 9, n. 2, pp. 200-211, 2013. 
[22] F. Sánchez, M. C. Martínez-Habibe, S. Díaz, N. O. Medina, J. Riaño y M. F. PaQui, "Biodiversidad en un campus universitario en la Sabana de Bogotá: inventario de plantas y tetrápodos", Centro de Museos. Bolet. Cient., vol. 19, pp. 186-203, 2015.

[23] J. Pacateque, P. Cruz, M, Aguilar y J. R. Cure, "Efecto de la alimentación vía bolsillo en etapas tempranas de desarrollo de Bombus atratus (Hymenoptera, Apidae)", Rev. Colomb. Entomol., vol. 38, pp. 343-346, 2012.

[24] G. Erdtman, "The acetolysis method: a revised description”, Sv. Bot. Tidskr. Lund., vol. 54, n. ${ }^{\circ} 4$, pp. 561$564,1960$.

[25] C. I. Silva et al., Catálogo polínico das plantas usadas por abelhas no Campus da USP de Ribeirão Preto, Ribeirão Preto: Holos Editor, 2014.

[26] Rede de Catálogos Polínicos online RCPol (2013). [En línea]. Disponible en: www.rcpol.org.br

[27] I. Montero y R. Tormo, "Análisis polínico de mieles de cuatro zonas montañosas de Extremadura", An. Asoc. Palinól. Leng. Esp., vol. 5, pp. 71-78, 1990.

[28] A. Maurizio y J. Louveaux, Pollens de plantes mellifères d'Europe, París: Union des Groupements Apicoles Français, 1965.

[29] J. D. Lobatón, J. R. Cure y M. T. Almanza, "Fenología $\mathrm{y}$ oferta floral de trébol rojo Trifolium pratense (Fabales: Leguminosae) en praderas de kikuyo (Penissetum clandestinum (Poales: Poaceae), como fuente de alimento para Bombus atratus (Hymenoptera: Apoidea) en Cajicá, Colombia”, Rev. Facul. Cien. Bás., vol. 8, pp. 18-27, 2012.

[30] A. Brian, "The pollen collected by Bumble-bee", J. Anim. Ecol., vol. 20, n. ${ }^{\circ}$ 2, pp., 191-194, 1951.

[31] E. Hennig y J. Ghazoul, "Plant-pollinator interactions within the urban environment", Persp. Plant Ecol., Evol. System., vol. 13, pp. 137-150, 2011.

[32] D. Rubio, "Disponibilidad, uso y preferencia por lo recursos florales en una comunidad de abejorros (Hymenoptera: Apidae: Bombus) en el páramo de Chingaza", tesis de maestría, Facultad de Ciencias, Universidad Nacional de Colombia, 2012.
[33] T. Roulston, J. Cane y S. Buchmann, "What governs protein content of pollen: pollinator preferences, pollen-pistil interactions, or phylogeny?", Ecol. Monogr., vol. 70, n. ${ }^{\circ} 4$, pp. 617-643, 2000.

[34] M. Hanley, M. Franco, S. Pichon, B. Darvill y D. Goulson, "Breeding system, pollinator choice and variation in pollen quality in British herbaceous plants", Funct. Ecol., vol. 22, pp. 592-598, 2008.

[35] C. Weiner, A. Hilpert, M. Werner, K. Linsenmair y N.O. Blüthgen N, "Pollen amino acids and flower specialization in solitary bees”, Apidol., vol. 41, pp. 476-487, 2010.

[36] S. Leonhardt y N. O. Blüthgen, "The same, but different: pollen foraging in honeybee and bumblebee colonies", Apidol., vol. 43, pp. 449-464, 2012.

[37] J. Free, Insect pollination of crops, Londres: Academic Press, 1970.

[38] M. C. Telleria, "Palynological analysis of food reserves found in a nest of Bombus atratus (Hym. Apidae)", Grana, vol. 37, pp. 125-127, 1998.

[39] E. Camillo y C. A. Garófalo, "Analysis of the niche of two sympatric species of Bombus (Hymenoptera, Apidae) in southeastern Brazil”, J. Trop. Ecol., vol. 5, pp. 81-92, 1989.

[40] L. B. De Faria, K. P. Aleixo, C.A. Garófalo, V. L Imperatriz y C. I. Da Silva, "Foraging of Scaptotrigona aff. depilis (Hymenoptera, Apidae) in an urbanized area: seasonality in resource availability and visited plants", Hindawi Publ. Corp. Psy., vol. 2012, 12 pp., 2012, doi: https://doi.org/10.1155/2012/630628

[41] K. P. Aleixo, L. B. De Faria, C. A. Garófalo, V. L. Imperatriz y C. I. da Silva, "Pollen collected and foraging activities of Frieseomelitta varia (Lepeletier) (Hymenoptera: Apidae) in an urban landscape", Sociobiol., vol. 60, n. ${ }^{\circ}$ 3, pp. 266-276, 2013. 